\title{
L'INDICE DE CONSOMMATION EST-IL, CHEZ LE PORC, LE TÉMOIN FIDĖLE DE L'EFFICACITÉ D'UNE RATION
}

PAR

\section{R. FÉRIER}

Station de recherches sur l'Élevage, Centre national de recherches zootechniques, Jouy-en-Josas

\section{INTRODUCTION}

Nous appelons Indice de Consommation le rapport de la quantité de nourriture consommée par un animal en croissance pendant une certaine période, au gain de poids vif qu'il a réalisé pendant le même temps. Le poids de l'animal est toujours exprimé en $\mathrm{kg}$ mais la quantité de nourriture peut être évaluée, selon la nécessité, en $\mathrm{kg}$ de matière brute, de matière sèche ou en unités énergétiques. Pour éviter toute erreur d'interprétation quand le doute est permis, nous employons la notation : Indice $(\mathrm{kg})$, Indice (U. F.), Indice (T. D. N.), etc...

La connaissance de ce quotient est très importante, au point de vue scientifique comme au point de vue pratique. Néanmoins, il nous est apparu à plusieurs reprises (9) que, pour le même matériel animal, ses variations en reflétaient pas toujours fidèlement les différences que peut présenter la valeur alimentaire de la ration, ou plus exactement, son aptitude à couvrir les besoins de l'animal (ce que nous appellerons désormais Efficacité, en employant ce terme dans son sens le plus large). Il y a là, pensons-nous, une source d'erreurs pour l'interprétation de certains résultats expérimentaux.

Nous avons procédé à quelques observations qui nous ont conduits :

à formuler certaines règles à observer lors d'expériences d'alimentation portant sur des animaux en croissance ;

à proposer une méthode d'interprétation des résultats obtenus, dans le cas où ces règles n'ont pas été observées. 


\section{I. - OBSERVATIONS}

\section{$I^{\circ}$ Le même aliment, distribué a des animaux semblables, peut ne pas donner le même indice de consommation}

Nous avons constitué 2 groupes d'animaux comparables : pendant un mois, ils ont réalisé le même gain de poids, ils ont consommé la même quantité de la même nourriture, ils ont eu le même indice de consommation (tableau I)

Ces animaux étaient donc semblables.

\section{TABLEAU I}

\section{Préexpérience $I$}

Lot $A \ldots \ldots \ldots \ldots \ldots \ldots$

\begin{tabular}{|c|c|c|c|c|}
\hline \multicolumn{2}{|c|}{ Poids moyen } & $\begin{array}{c}\text { Gain } \\
\text { moyen } \\
\text { par ani- } \\
\text { mail en } 4 \\
\text { semaines. }\end{array}$ & $\begin{array}{c}\text { Consom- } \\
\text { mation } \\
\text { par ani- } \\
\text { mal en } 4 \\
\text { semaines. }\end{array}$ & $\begin{array}{c}\text { Indice de } \\
\text { consom- } \\
\text { mation } \\
\text { moyen. }\end{array}$ \\
\hline$\overline{\mathrm{kg}}$ & $\overline{\mathrm{kg}}$ & $\overline{\mathrm{kg}}$ & $\mathrm{kg}$ & $\mathrm{kg}$ \\
\hline 5 & 66 & 14,3 & 57, & 3,99 \\
\hline 49 & 63,3 & $\mathrm{I}_{4,3}$ & 56,8 & 3,96 \\
\hline
\end{tabular}

Sans changer de nourriture, nous les avons soumis, pendant 6 semaines au rationnement suivant : les rations furent réduites d'environ $20 \%$ pour le lot A par rapport au lot B qui était normalement alimenté en 3 repas, selon l'appétit des sujets.

Les résultats sont présentés dans le tableau II :

\section{TABlEAu II}

\section{Expérience $I$}

\begin{tabular}{|c|c|c|c|c|c|}
\hline & $\begin{array}{l}\text { Poids } \\
\text { moyen } \\
\text { départ. } \\
\frac{\text { ko }}{}\end{array}$ & $\begin{array}{c}\text { Gain } \\
\text { moyen } \\
\text { par ani- } \\
\text { mal en } 6 \\
\text { semaines. } \\
- \\
\mathrm{kg}\end{array}$ & $\begin{array}{c}\text { Gain } \\
\text { moyen } \\
\text { quotidien. } \\
\frac{-}{\mathrm{kg}}\end{array}$ & $\begin{array}{l}\text { Consom- } \\
\text { mation } \\
\text { par ani- } \\
\text { mal en } 6 \\
\text { semaines. } \\
- \\
\mathrm{kg}\end{array}$ & $\begin{array}{l}\text { Indice de } \\
\text { ronsom. } \\
\text { mation } \\
\text { moyen, } \\
\overline{\mathrm{kg}}\end{array}$ \\
\hline$\ldots \ldots \ldots \ldots \ldots$ & 66,3 & 21,3 & 0,508 & 109 & $5, \mathrm{I} \mathrm{I}$ \\
\hline ot $B \ldots \ldots \ldots \ldots \ldots \ldots$ & 63,3 & 28,7 & 0,682 & I 35 & 4,69 \\
\hline
\end{tabular}

Nous voyons ainsi que l'indice de consommation du lot A (rationné) est de ro \% supérieur à celui du lot $\mathrm{B}$. Le même aliment peut donc donner, sur des animaux semblables, des indices de consommation sensiblement différents. La nourriture étant identique dans les 2 lots, nous pouvons affirmer que la différence entre des indices obtenus sur des animaux semblables n'indique pas, ipso facto, une différence entre les efficacités des rations employées. 


\section{$2^{\circ}$ Deux aliments d'efficacité différente peuvent donner le même indice}

Nous avons voulu procéder à la contre-épreuve de l'expérience précédente.

Deux groupes d'animaux comparables ont été constitués, après une épreuve préalable de 4 semaines (tableau III) :

TABLEAU III

Préexpérience II

Lot A. . . W .

\begin{tabular}{|c|c|c|c|c|}
\hline \multicolumn{2}{|c|}{ Poids moyen } & $\begin{array}{l}\text { Gain } \\
\text { moyen } \\
\text { pendant } \\
28 \text { jours }\end{array}$ & $\begin{array}{c}\text { Consom- } \\
\text { mation } \\
\text { moyenne } \\
\text { pendant } \\
28 \text { jours. }\end{array}$ & $\begin{array}{c}\text { Indice d } \\
\text { consom } \\
\text { mation } \\
\text { moyen. }\end{array}$ \\
\hline 21, & $3^{6}$ & 14,1 & & \\
\hline 20,6 & 34,3 & r 3,7 & 43,5 & $3, \mathrm{I}$ \\
\hline
\end{tabular}

Nous avons alors distribué à ces animaux, pendant 5 semaines, deux aliments dont les protéines possédaient une valeur manifestement très différente, mais, par un rationnement approprié, nous avons maintenu nos animaux à la même vitesse de croissance.

Les formules utilisées étaient les suivantes :

\section{A}

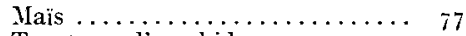

Tourteau d'arachide $\ldots \ldots \ldots \ldots \ldots$. 20

Mélange minéral............ 3
$B$

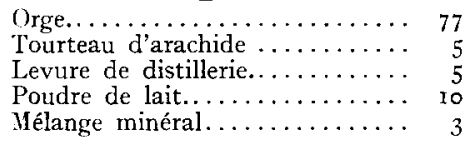

Les résultats obtenus figurent dans le tableau IV :

TABLEAU IV

Expérience II

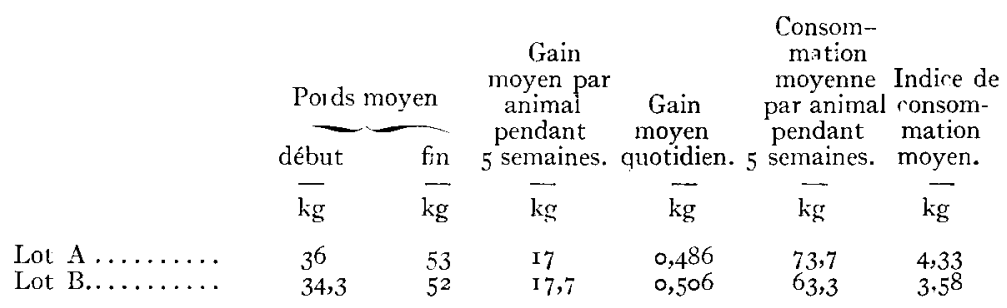

Les animaux ayant sensiblement la même croissance, leurs besoins étaient identiques. Or, nous constatons que, pour les couvrir, il a fallu une quantité plus grande de l'aliment A que de l'aliment B. Nous powvons dire que l'efficacité de l'aliment $A$ est inférieure à celle de l'aliment $B$.

Par la suite, en réglant convenablement l'alimentation des animaux, nous avons obtenu sensiblement le même indice pour les deux lots, au cours d'une période de 6 semaines (tableau V) : 
TABLEAU V

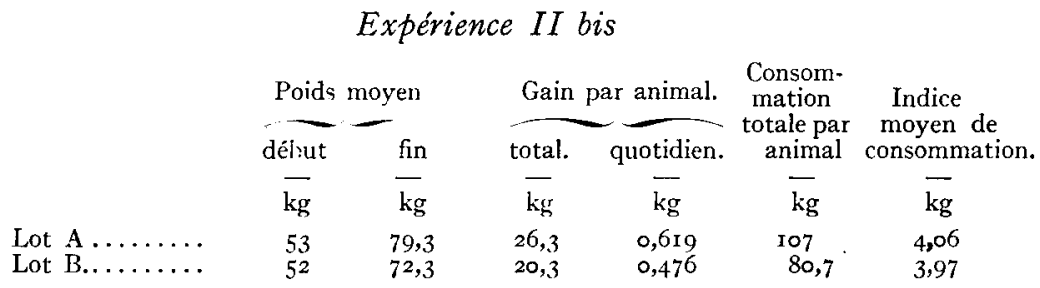

Donc, l'identité des indices n'implique pas, ipso facto, l'identité des efficacités.

Nous avons ainsi montré que les variations de l'indice de consommation ne reflétaient pas toujours les variations de l'efficacité de la ration, dont, par conséquent, l'indice de consommation ne constitue pas le témoin fidèle.

\section{II. - DISCUSSION}

Certaines considérations d'ordre théorique peuvent nous permettre d'expliquer le phénomène observé :

Considérons un animal en croissance recevant un aliment déterminé, à la dose journalière $T$. Cette ration couvre, d'une part, le besoin d'entretien qui correspond à la fraction $\mathrm{E}$ de la ration, et d'autre part, le besoin de production, qui représente la différence. Soit $G$ le gain quotidien, nous pouvons appeler $P$ le besoin de croissance rapporté à l'unité de gain, tel que :

$$
\mathrm{T}=\mathrm{E}+\mathrm{PG} \text {. }
$$

L'indice de consommation I se définit par le rapport :

$$
\mathrm{I}=\frac{\mathrm{T}}{\mathrm{G}}=\frac{\mathrm{E}+\mathrm{PG}}{\mathrm{G}}
$$

ou

$$
\mathrm{I}=\frac{\mathrm{E}}{\mathrm{G}}+\mathrm{P} .
$$

La vitesse de croissance est sous la dépendance du volume de l'ingestion, lequel est fonction de l'appétence de l'aliment ou de l'appétit de 1'animal. Si $G$ varie dans de faibles limites, $E$ et $P$ restent sensiblement constants, et I varie en sens inverse de $\mathrm{G}$.

Dans l'expérience $\mathrm{I}$, quand nous avons diminué les rations du lot $\mathrm{A}$ donc la vitesse de croissance - nous avons augmenté l'indice de consommation. Dans l'expérience II bis, pour élever l'indice de consommation du lot $\mathrm{B}$ au niveau de celui du $\operatorname{lot} A$, nous avons dû diminuer les rations du lot $B$ et, par conséquent, la vitesse de croissance des animaux de ce lot. L'équation (I) permet d'expliquer quantitativement ces résultats :

Dans l'expérience I, par exemple, nous savons par l'analyse que I $\mathrm{kg}$ de l'aliment équilibré utilisé est égal à une unité fourragère. 
Nous avons, pour le lot $B$ :

poids moyen des animaux pendant 1'expérience : $78 \mathrm{~kg}$, d'où $\mathrm{E}=0,97$ U. F. d'après les tables (9)

$$
\mathrm{P}=\mathrm{I}-\frac{\mathrm{E}}{\mathrm{G}}=4,69-\frac{0,97}{0,682}=3,27 \text {. }
$$

A partir de ces données, essayons de prévoir l'indice de consommation théorique du lot A :

poids moyen pendant l'expérience : $77 \mathrm{~kg}$ d'où $\mathrm{E}=0,96$.

Les animaux étant comparables, $\mathrm{P}$ a la même valeur pour les deux lots:

$$
\mathrm{I}^{\prime}=\frac{0,96}{0,508}+3,27=5, \mathrm{I} 6
$$

Or, la valeur constatée expérimentalement est de 5,II. La concordance est donc satisfaisante entre les résultats expérimentaux et la valeur obtenue par le calcul.

\section{CONCLUSIONS}

I. - Cette influence de la vitesse de croissance sur la valeur de l'indice de consommation étant montrée, et, pensons-nous, expliquée, il nous semble 1ogique, pour éliminer ce facteur de variation dans des expériences d'alimentation, de maintenir par un rationnement approprié, les animaux à des niveaux de croissance très voisins.

Ainsi peut-on comparer quantitativement avec rigueur l'efficacité des différents aliments utilisés. Dans ce cas, on peut considérer que le rapport des indices de consommation est égal à l'inverse du rapport des efficacités.

Il peut arriver que la valeur de ce rapport ne soit pas constante et dépende de la vitesse de croissance imposée aux animaux, car les besoins qualitatifs sont parfois différents dans l'entretien et dans la croissance. Il importe donc de préciser, dans l'expression des résultats, les conditions dans lesquelles la mesure a été effectuée.

A ce propos, il est regrettable que nous n'ayons pas la possibilité d'agir sur l'appétit de l'animal, et de déterminer ainsi librement le niveau de croissance auquel nous désirons procéder à la comparaison des rations. Des recherches sur ce point seraient susceptibles de nous apporter des renseignements extrêmement utiles, car, pour l'instant, nous sommes obligés de régler la croissance des animaux sur la plus lente, et il peut en résulter un certain nombre d'inconvénients.

La méthode qui consiste à nourrir les animaux ad libitum et à observer leur croissance et leur indice de consommation, autorise des conclusions intéressantes, surtout du point de vue pratique, sur la valeur alimentaire globale de la ration, mais elle ne renseigne pas sur le facteur (ou les facteurs) auquel il convient d'attribuer l'effet constaté. Sur ce point, nos critiques rejoignent celles de Carroli (4), de Mitchell et Beadlés (I4) et de Brody (3). 
Certains auteurs, tels que Mitcheli, et BeAdies (I4) préconisent la méthode qui consiste à distribuer la même quantité de nourriture aux animaux des différents lots (paired-feeding method), et à en observer les effets sur la croissance et l'indice de consommation. On obtient alors un classement correct des différents régimes, puisque l'influence de l'appétit de l'animal est éliminée, mais ce n'est là qu'un renseignement d'ordre qualitatif. Il est suffisant lorsqu'on s'inquiète de la présence ou de la déficience d'un élément bien déterminé (oligoélément, vitamine, acide aminé, etc...). Mais Mitchell et HAMIIToN (I6), disent : "Une telle méthode est admirablement adaptée au problème de savoir quel est le meilleur des deux régimes, mais elle ne permet pas de mesurer avec précision de combien l'un est supérieur à l'autre ".

Dans ce cas, ils préconisent la méthode que nous avons décrite, que MITCHELI. (I3) considère comme l'idéale (body-weight equalization) et qu'il a utilisée, ainsi que d'autres auteurs tels que KLEIBER (8) et HAMILTON (5).

II. - Le rôle considérable que joue la vitesse de croissance sur l'utilisation de la notrriture met en relief toute l'importance que présente le volume de 1'ingestion, donc l'appétit de l'animal et l'appétence de la ration.

Morrisson ( $\mathrm{I} 7$ ) dit très justement : " Dans la plupart des cas, la bonne ration est supérieure du point de vue économique, parce qu'elle provoque la plus grande consommation et la plus grande production ". Mais il ne semble pas y avoir de liaison entre la sapidité de la ration et son efficacité, comme l'ont indiqué Mitchel. (I5) et, très récemment, Aschkenasy-LELU (I). Il paraît donc intéressant de mesurer la "palatabilité » de la ration au cours d'une épreuve distincte.

III. - Quand les précautions sus-indiquées n'ont pas été prises, nous avons imaginé une méthode qui permet une interprétation des résultats expérimentaux.

Comme la plupart des méthodes faisant intervenir le calcul en biologie, elle ne saurait prétendre remplacer la méthode directe, mais, malgré son manque de précision, nous pensons qu'elle peut rendre certains services :

Soient 2 lots d'animaux soumis à 2 régimes alimentaires différents, l'un témoin $t$, l'autre expérimental $e$. Supposons que leurs croissances aient été différentes ( $G t$ et $\mathrm{G} e$ ) : nous savons que la comparaison des indices de consommation obtenus ne permet pas de conclusion rigoureuse quant à l'efficacité relative de ces deux régimes.

Mais imaginons un lot témoin $t^{\prime}$ dont la croissance aurait été la même que celle du lot expérimental $e$. La comparaison des indices de consommation de $t^{\prime}$ et de $e$ renseignerait de façon précise sur les valeurs relatives de l'efficacité des deux régimes.

Essayons de calculer 1'indice It' de ce lot fictif :

L'équation :

$$
\mathrm{I} t=\frac{\mathrm{E} t}{\mathrm{G} t}+\mathrm{P} t
$$


dans laquelle :

It et $\mathrm{G} t$ sont déterminés expérimentalement, $\mathrm{E} t$, exprimé en $\mathrm{kg}$ de 1'aliment témoin, peut être calculé d'après les tables (8), si nous connaissons la valeur énergétique de cet aliment, nous permet de calculer $\mathrm{P} t$, lequel, si la différence $\mathrm{G} t$-Ge n'est pas excessive, est voisin de $\mathrm{P} e$.

Pour le lot témoin $t^{\prime}$, nous avons :

$$
\mathrm{I} t^{\prime}=\frac{\mathrm{E} t^{\prime}}{\mathrm{G} t^{\prime}}+\mathrm{P} t^{\prime}
$$

Par hypothèse, $\mathrm{G} t^{\prime}=\mathrm{G} e$; or, nous connaissons $\mathrm{G} e$.

Le poids moyen des animaux du lot $t^{\prime}$ est le même que celui des animaux du lot $e$, puisque le poids au départ est comparable et que $\mathrm{G} t^{\prime}=\mathrm{G} e$. Les tables nous permettront de calculer $\mathrm{E} t$ ', comme nous avons calculé $\mathrm{E} t$.

$L^{\prime}$ indice $I \mathrm{t}$ ' ainsi calculé représente l'indice d'un lot témoin dont la croissance aurait été la même que celle du lot expérimental.

Dans ces conditions, le rapport $\frac{\mathrm{I} t^{\prime}}{\mathrm{I} e}$ peut être considéré comme l'inverse du rapport des efficacités de 1'aliment témoin et de l'aliment expérimental.

Les erreurs que l'on peut commettre en utilisant cette méthode, peuvent provenir des causes suivantes:

I. - Les valeurs du besoin d'entretien que fournissent les tables, sont des moyennes portant sur un très grand nombre de sujets. Elles ne s'appliquent à la population limitée et choisie constituant le matériel expérimental qu'avec une certaine approximation.

2. - Ces valeurs, exprimées en unités énergétiques, ne concernent qu'une ration équilibrée. Or, quand les sujets des lots témoins ( $t$ et $\left.t^{\prime}\right)$ sont nourris avec un aliment équilibré et bien connu, tel que notre aliment standard (II), on peut valablement utiliser les renseignements donnés par ces tables. Mais, quand il s'agit de comparer entre enx des aliments mal connus, dont l'équilibre est problématique, il est incorrect d'utiliser les valeurs énergétiques déduites de 1'analyse chimique pour calculer, à partir des tables, les besoins du lot pris comme témoin.

3. - Le besoin de croissance rapporté à l'unité de gain, $\mathrm{P}$, est supposé constant pour un poids vif donné. Ceci n'est vrai, pour le même matériel animal, que si les croissancessont les mêmes, comme le souligne BRODy (2). Mc MEEKAN (I2) a montré qu'une élévation de la vitesse de croissance s'accompagnait d'une élaboration plus grande de tissus adipeux et se 'traduisait par une augmentation de $P$. En supposant $P$ constant, nous commettons une erreur d'autant plus grande que la différence des vitesses de croissance est plus grande.

Ces diverses considérations montrent qu'il convient d'utiliser ce calcul avec beaucoup de prudence et que la technique précédemment proposée, beaucoup plus rigoureuse, s'impose chaque fois qu'il est possible d'y recourir. 
L'expérience II et II bis (tableaux IV-V) nous permet d'éprouver notre méthode. En effet, nous avions comparé directement l'efficacité des régimes A et $\mathrm{B}$ dans l'expérience II. Le rapport des indices était :

$$
\frac{\mathrm{IA}}{\mathrm{IB}}=\frac{4,33}{3,5^{8}}=\mathrm{I}, 20 .
$$

Voyons ce que donne notre méthode (expérience II bis) :

Le poids moyen des animaux, pendant la période expérimentale, a été :

$66 \mathrm{~kg}$ pour le lot A

$62 \mathrm{~kg}$ pour le lot $B$

La valeur fourragère de $A$ et $B$ étant égale à $x \mathrm{U}$. F. par $\mathrm{kg}$ les tables nous donnent, pour le besoin d'entretien évalué en aliment " $\mathrm{A}$ »:

$$
0,87 \mathrm{~kg} \text { pour le lot } \mathrm{A}
$$

Appelons A' le lot fictif que nous avons imaginé. Toujours d'après les tables, nous avons :

$$
\mathrm{E}_{\mathrm{A}}{ }^{\prime}=0,84 \mathrm{~kg} \text { de 1'aliment } \mathrm{A}
$$

L'équation (I) nous permet de calculer $\mathrm{P}$ :

$$
\mathrm{P}=4,06-\frac{0,87}{0,6 \mathrm{r} 9}=2,65
$$

et

$$
\mathrm{IA}^{\prime}=\frac{0,84}{0,476}+2,65=4,4 \mathrm{r} .
$$

Comparons cet indice à celui mesuré expérimentalement sur le lot B :

$$
\frac{4,4 \mathrm{I}}{3,97}=\mathrm{I}, \mathrm{II} \text {. }
$$

Le rapport des indices expérimentaux obtenus dans l'expérience II était, rappelons-le :

$$
\frac{4,33}{3,58}=\mathrm{I}, 20 \text {. }
$$

La concordance n'est pas absolue. Nous n'y prétendions pas; mais le calcul

\begin{tabular}{|c|c|c|c|c|}
\hline Lot. & $\begin{array}{l}\text { lait par } \\
\mathrm{kg} \text { d'orge } \\
\mathrm{kg} \text {. }\end{array}$ & $\begin{array}{l}\text { Consommation } \\
\text { moyenne } \\
\text { quotidienne. }\end{array}$ & $\begin{array}{c}\text { Gain } \\
\text { moyen } \\
\text { quotidien. }\end{array}$ & $\begin{array}{l}\text { Indice de } \\
\text { consommation. }\end{array}$ \\
\hline & $\therefore$ & $\bar{U} . \bar{F}$ & $\overline{\mathrm{kg}}$ & U.F. \\
\hline I. $\ldots \ldots \ldots \ldots \ldots \ldots \ldots \ldots \ldots \ldots \ldots$ & $\circ$ & $\mathrm{I}, 57$ & 0,244 & 6,50 \\
\hline $2 \ldots \ldots \ldots \ldots \ldots \ldots \ldots \ldots \ldots \ldots$ & 0,5 & 2,15 & 0,473 & 4,54 \\
\hline  & I & 2,44 & 0,594 & 4,09 \\
\hline ....... & $\mathrm{I}, 5$ & $2,7 \mathrm{I}$ & 0,706 & 3,84 \\
\hline $5 \ldots \ldots \ldots \ldots \ldots \ldots \ldots \ldots \ldots, \ldots$ & 2 & $2,8 \mathrm{I}$ & 0,706 & 3.95 \\
\hline
\end{tabular}
nous permet de dire qu'il existe une sensible différence d'efficacité entre les deux aliments, en faveur de l'aliment $B$, affirmation que le simple examen des résultats de l'expérience II bis (tableau V) ne nous permettait pas de produire.

Un autre exemple particulièrement typique nous est fourni par la littérature. Etudiant le taux optimum de lait écrémé à mélanger à l'orge pour des porcs en croissance, JESPERSEN (6) a obtenu les résultats suivant : 
Autrement dit, en élevant la proportion de lait écrémé dans la ration, on augmente la consommation quotidienne (donc la vitesse de croissance) et l'on abaisse 1'indice de consommation. Cet abaissement est-il dû à un accroissement d'appétence ou à un accroissement'd'efficacité de la ration ? Le simple examen des résultats expérimentaux ne permet pas de le dire.

Notre méthode, au contraire, permet de distinguer approximativement l'influence de ces deux facteurs : appétence et efficacité.

Prenant le lot 4 (dont la ration paraît la mieux équilibrée) comme lot témoin, nous avons calculé, en utilisant les normes de JESPERSEN (7), les indices I' de lots témoins fictifs dont les vitesses de croissance seraient respectivement réglées sur celles des lots réels $I, 2,3,5$.

Nous avons obtent le tablean suivant :

\begin{tabular}{|c|c|c|c|}
\hline Lot. & Indice réel $\mathrm{I}$. & Indice fictil I & Rapport $\frac{\mathrm{I}^{\prime}}{\mathrm{I}}$ \\
\hline- & - & - & - \\
\hline 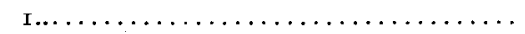 & 6,50 & 5,55 & 0,85 \\
\hline 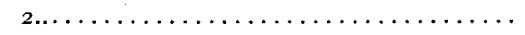 & 4,54 & 3,88 & 0,85 \\
\hline $3 \ldots \ldots \ldots \ldots \ldots \ldots \ldots \ldots \ldots \ldots$, & 4,09 & 3,88 & 0,95 \\
\hline 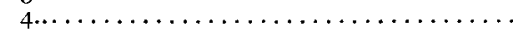 & 3,84 & 3,84 & I \\
\hline 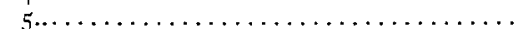 & 3,95 & 3,84 & 0,97 \\
\hline
\end{tabular}

Alors que l'indice de consommation variait dans de très larges proportions (de 6,5 à 3,84, soit de $I, 7$ à I), pour des animaux nourris ad libitum, il aurait vraisemblablement varié seulement de $I_{5} \%\left(0,85^{-I}\right)$ environ si la même vitesse de croissance leur avait été imposée.

Les variations de l'indice de consommation sont ici en sens inverse des variations de l'efficacité, mais leurs amplitudes sont bien différentes, le lait intervenant dans une plus large mesure comme facteur de sapidité que comme facteur d'équilibre de la ration.

\section{RESUME}

I. - Les variations de l'indice de consommation, observées sur le même matériel animal, ne reflètent pas fidèlement les variations de l'efficacité de la ration.

2. - Dans ces conditions, il importe de mesurer l'indice de consommation dans des conditions identiques de croissance, si l'on veut comparer avec rigueur l'efficacité de plusieurs rations.

3. - Si 1'aspect qualitatif de la comparaison est suffisant pour le but recherché, la distribution de rations quantitativement égales permet de les classer selon leur efficacité.

4. - Au cas où ces précautions n'auraient pas été prises, il est possible de procéder - quoique avec moins de précision - à cette comparaison, en imaginant un lot témoin fictif dont la croissance serait la même que celle du lot expérimental et en calculant, à partir des données expérimentales et des tables, quel aurait été son inđice de consommation. 
5. - L'appétence d'un aliment joute un rôle considérable dans la "valeur alimentaire " globale de celui-ci, puisqu'elle agit sur la vitesse de croissance, par l'intermédiaire de l'ingestion plus ou moins importante qu'elle provoque, Il est souvent intéressant de la mesurer au cours d'une épreuve spéciale.

\section{BIBLIOGRAPHIE}

(I) Aschkenasy-Lelu (P.). - L'ingestion alimentaire en fonction de la composition du régime. Application à la régulation du besoin azoté chez le rat. Ann. Nutr. et Alim., $\mathrm{V}, 4-5$, p. $454,195^{2}$.

(2) Brody (S.). - Nutrition. Ann. Rev. Biochem., IV, p. 383-412, I935.

(3) BRODY (S.). - Bioenergetic and growth. (Reinhold publish. Corp. N. Y.), p. 740-791, I945.

(4) Carroll (W. E.). - Group feeding as a method of livestock experimentation. Amer. Soc. An. Prod., Proc. $23^{\circ}$ meet., p. 34*44, r93o.

(5) Hamilton (T.S.). - The growth, activity, and composition of rats fed diets balanced and unbalanced with respect to protein. J. Nutrition, 17, p. 565 , I939.

(6) Jespersen (J.). - Iste Beretning om de af Forsglaboratoriet og De samvirkende danske Andels-Svineslagterier foranstaltede Fodringsforsg med Svin Forsg med Skummetmaelk. I280 Beretning fra Forcglaboratoriet, 1928 .

(7) Jespersen (J.). - Normes pour les besoins des chevaux, porcs, poules. Ve Congrès international de Zootechnie, p. 33-40, 1949.

(8) Kleiber (M.), Boelter (M. D. D.) et Greenberg (D. M.). - Fasting catabolism and food utilization of calcium-deficient rats. J. Nutrition, 19, p. 51 7, 1940.

(9) Leroy (A. M.). - Le Porc (Ed. Hachette), i937.

(Io) Leroy (A. M.) et FÉvrier (R.). - Influence du mode de distribution des repas sur l'utilisation de la nourriture par le porc. Ann. Agron., 5, p. 765-770, r 947 et 4, p. $605^{-}$ $6 \mathrm{II}, \mathrm{I} 949$.

(I I) Leroy (A. M.) et FÉvrier (R.). - Étude des aptitudes économiques et de leur transmission chez les différentes souches et races porcines. Ann. Agron., 6, p. 925-950, I949.

(I2) Mc Meekan (C. P.). - Growth and development in the bacon pig with special reference to carcase quality. $J$. Agric. Sci., 30, p. 276, 1940.

(13) Mitcheli (H. H.). - The paired-feeding method : its value and limitations in livestock experimentation. Amer. Soc. An. Prod., Proc. 23० meeting, p. 63-73, I930.

(I4) Mitchell (H. H.) et Beadles (J. R.). - The paired-feeding method in nutrition experiments and its application to the problem of cystine deficiencies in food proteins. J. Nutrition, 2, p. 225-24.3, 1930.

(I5) Mitchell (H. H.). - Balanced diets net energy values and specific dynamic effects. Science, 80, p. $55^{8}$, 1934 .

(i6) Mitchell (H. H.) et Hamilton (T. S.). - The balancing of rations with respect to protein. Amer. Soc. An. Prod., Proc. 28 meeting, p. 24I-252, 1935.

(i 7 ) Morrisson (F. B.). - Amer. Soc. An. Prod. Proc. 26 meeting, p. 27, 1933, in (2). 